\title{
La stratégie européenne en physique des particules
}

Gautier Hamel de Monchenault(1) (gautier.hamel-de-monchenault@cea.fr)

et Laurent Vacavant ${ }^{(2)}$ (laurent.vacavant@cnrs.fr)

(1) Département de physique des particules, Irfu, CEA/Saclay,

91191 Gif-sur-Yvette Cedex

CERN, Porte-parole adjoint de l'expérience CMS

(2) Centre de physique des particules de Marseille, 163 avenue de Luminy, 13288 Marseille Cedex 09

Directeur adjoint scientifique pour la physique des particules et la physique hadronique (CNRS/IN2P3)

La communauté européenne

de physique des particules

a actualisé en 2020 sa feuille

de route, dont la mise à jour a lieu

environ tous les sept ans depuis

2005, sous l'égide du CERN,

l'Organisation européenne

pour la recherche nucléaire.

La mise à jour de 2020 formule

des recommandations

sur les priorités scientifiques

et les futurs objectifs

de la physique des particules

dans un contexte mondial,

sur les instruments qui offrent

le meilleur potentiel pour atteindre

ces objectifs, et sur les technologies

qui doivent être développées

pour construire ces instruments.

L'ambition de cet article est

d'apporter un éclairage

sur ces recommandations.
La mise à jour de la stratégie européenne en physique des particules est un processus rigoureux et approfondi qui a engagé la communauté des physiciens pendant près de deux ans [1]. Dans un premier temps, celle-ci s'est exprimée : cent soixante contributions, individuelles ou institutionnelles, ont été reçues à la fin de 2018. Puis, la communauté s'est réunie lors d'un symposium en mai 2019 à Grenade, Espagne. Les discussions y étaient organisées selon huit thématiques sous la supervision d'un panel d'experts, dont le rôle a été par la suite de synthétiser l'ensemble des contributions, discussions et conclusions dans un document, le Physics Briefing Book [2], publié en septembre 2019.

Ces travaux ont servi de base scientifique aux réflexions du Groupe de stratégie européen (ESG) en charge de préparer le document de mise à jour de la stratégie. L'ESG comprenait des représentants des vingt-trois états membres du CERN ${ }^{(a)}$, les directeurs des dix principaux laboratoires de physique des particules en Europe ${ }^{(b)}$, ainsi qu'un certain nombre d'invités et d'observateurs. La rédaction du document a eu lieu en janvier 2020 à Bad Honnef, en Allemagne.

Le document final [3] a été présenté au Conseil du CERN en mars, puis approuvé à l'unanimité par celui-ci en juin 2020. L'implémentation concrète des recommandations de la mise à jour de la Stratégie 2020 est confiée au CERN, sous la responsabilité de sa directrice générale, Fabiola Gianotti.

\section{La physique des particules après dix années d'exploitation du LHC}

La physique des particules vise à étudier les interactions entre les constituants fondamentaux de la matière. Le cadre théorique est le modèle standard (MS) de la physique des particules, une théorie quantique et relativiste des particules de matière (quarks et leptons) interagissant par l'échange de porteurs de force (bosons vecteurs). Le MS repose sur deux piliers : la théorie quantique des interactions nucléaires fortes, connue sous le nom de chromodynamique quantique (QCD), et la théorie électrofaible (EW), qui unifie la théorie quantique de l'électromagnétisme (QED) et la théorie de Fermi des interactions nucléaires faibles.

Le MS est une théorie extraordinairement prédictive lorsqu'on connait la valeur d'une vingtaine de paramètres libres. La plupart de ces valeurs proviennent de décennies de mesures de précision, en particulier auprès du collisionneur électronpositon LEP du CERN dans les années 1990, et du collisionneur proton-antiproton Tevatron du Fermilab (États-Unis) dans les années 1990 et 2000. De nombreux paramètres du MS sont intimement liés par des relations quantiques : l'un des triomphes du MS a été la prédiction de la masse du quark top à partir des mesures du LEP, peu avant sa découverte au Tevatron, en 1994.

(a) Dont Reynald Pain, directeur de l'IN2P3, représentant la France.

(b) Dont Achille Stocchi, directeur de l'IJCLab à Orsay, et Anne-Isabelle Étienvre, directrice du CEA/Irfu à Saclay. 
La pierre angulaire de la théorie est le phénomène de brisure spontanée de la symétrie électrofaible (EWSB), à l'origine d'une transition de phase de l'Univers primordial à l'issue de laquelle les bosons vecteurs (W et $\mathrm{Z}$ ) de l'interaction faible ont acquis leur masse, ainsi par ailleurs que les quarks et les leptons.

La découverte du boson de Higgs en 2012 [4] par les expériences ATLAS et CMS au Grand collisionneur de hadrons (LHC) du CERN (fig. 1), plus de quarante ans après la prédiction de son existence, confirme le mécanisme proposé en 1964 par Robert Brout, François Englert, Peter Higgs (BEH) et d'autres chercheurs comme à l'origine de l'EWSB. Elle consacre le MS comme l'une des théories les plus abouties de l'histoire des sciences.

Depuis 2012,ATLAS et CMS ont mesuré avec toujours plus de précision les propriétés du boson de Higgs, sans parvenir à mettre en défaut les prédictions du MS.

Bien que conforté par l'expérience, le MS laisse en suspens des questions fondamentales, notamment celle de la "matière noire", une substance gravitante invisible, bien plus abondante que la matière ordinaire à l'échelle cosmologique, mais dont la nature est inconnue.

L'énigme la plus profonde du MS concerne le boson de Higgs lui-même. La valeur de sa masse est précisément celle qui assure une stabilité quasi éternelle du vide, sans qu'il soit nécessaire de faire appel à de la nouvelle physique. Or, cette masse résulte d'un ajustement incroyablement fin entre des termes qui croissent d'autant plus que le domaine de validité du MS s'étend.

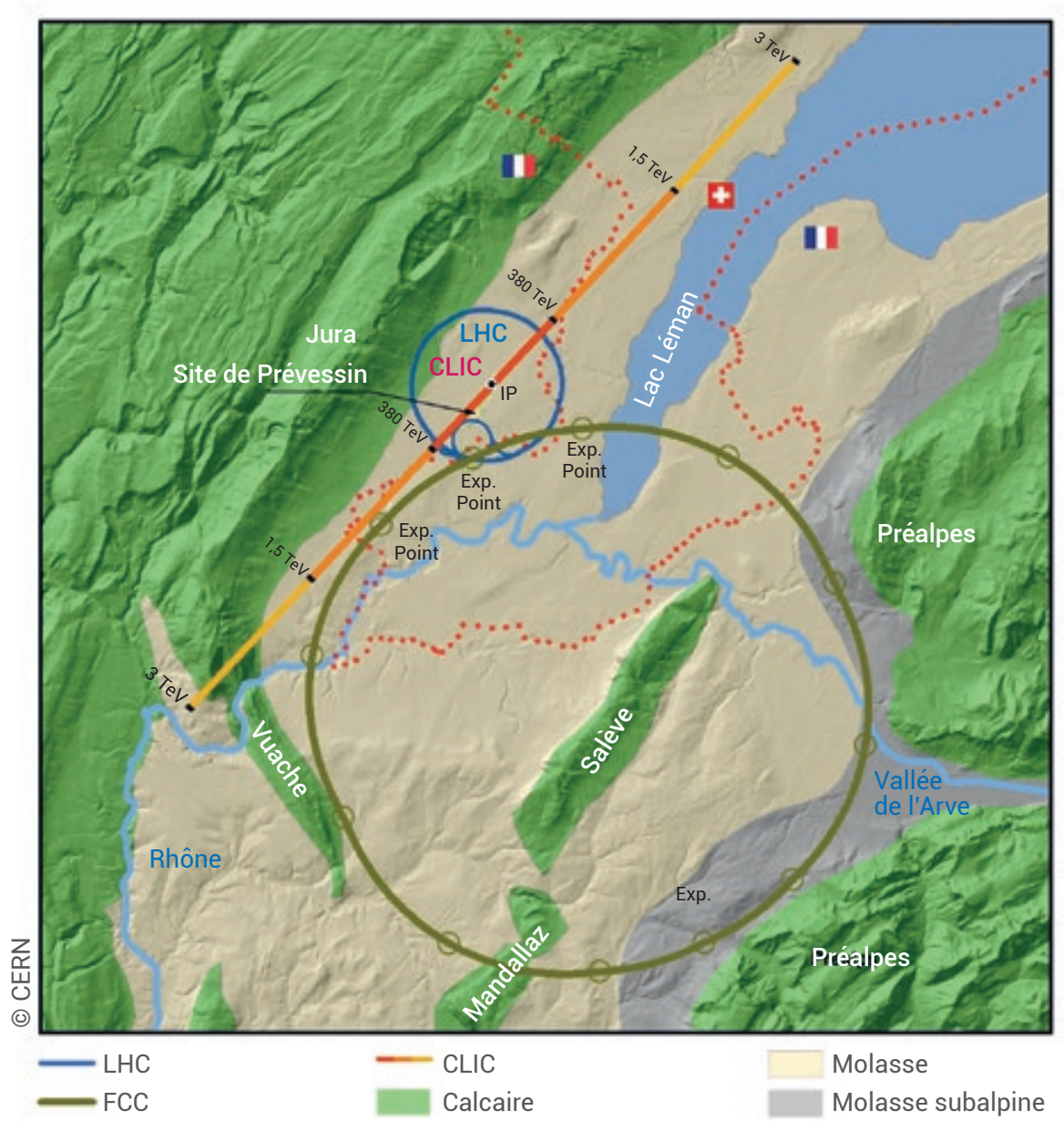

1. Vue de la région du CERN, près de Genève. La frontière franco-suisse est représentée en points rouges. Le Grand collisionneur de hadrons LHC, représenté par un cercle bleu en trait plein, est installé dans un tunnel de 27 km de circonférence à environ $100 \mathrm{~m}$ de profondeur, principalement dans le pays de Gex. Le petit cercle contenu représente le tunnel de $7 \mathrm{~km}$ de circonférence du Supersynchrotron à protons SPS, qui sert d'injecteur au LHC. Le grand cercle en trait continu kaki englobant le massif du Salève représente le tunnel du projet FCC, dont la circonférence est trois fois supérieure à celle du LHC. La ligne droite en trait continu orange le long du Jura et du lac Léman représente le tunnel du CLIC, un projet de collisionneur électron-positon linéaire de très haute énergie.

Ce problème, dit de la "naturalité " de la masse du boson de Higgs, a motivé de nombreux modèles théoriques qui prolongent le MS en postulant de nouvelles interactions, de nouveaux phénomènes ou l'existence de nouvelles particules. Or, rien de tout cela n'a été observé à ce jour au LHC. Le problème reste donc entier.

\section{Les principales recommandations de la Stratégie 2020}

C'est pourquoi la Stratégie réaffirme en préambule son fort soutien à l'exploitation optimale du potentiel de physique du LHC, notamment à la phase à haute luminosité
(HL-LHC). Celle-ci débutera en 2027 pour une période de dix ans, avec l'objectif de multiplier par dix la quantité de données, offrant ainsi une sensibilité accrue aux tests du MS et éventuellement à l'observation d'une nouvelle physique. Cela implique de tout mettre en œuvre pour assurer le plein succès de la mise à niveau du complexe d'accélérateurs et des expériences. Ce programme progresse bien, notamment dans les laboratoires de l'IN2P3 et de l'Irfu, selon un calendrier qui pourrait être légèrement révisé pour tenir compte de retards dus à la pandémie de Covid-19.

Pour la suite, la Stratégie considère l'étude détaillée du boson de Higgs comme la première priorité de la discipline. 
$>>$

Anticipant l'état des connaissances au terme de l'exploitation du HL-LHC, soit une précision de 2 à 10\% sur l'intensité des interactions entre le boson de Higgs et les bosons $\mathrm{W}$ et $\mathrm{Z}$ ou les fermions les plus massifs, elle identifie un collisionneur électron-positon de type "usine à Higgs " ("Higgs Factory" en anglais) comme la future grande machine pour prendre le relais et permettre d'atteindre une précision au moins dix fois meilleure.

Quatre projets d'usines à Higgs (fig. 2) sont considérés. Deux sont des collisionneurs électron-positon linéaires : l'International Linear Collider (ILC) au Japon et le Compact Linear Collider (CLIC) au CERN. Les deux autres sont circulaires : le Future Circular $\mathrm{e}^{+} \mathrm{e}^{-}$Collider (FCC-ee) au CERN et le Circular Electron Positron Collider (CEPC) en Chine.

Parmi ces projets, l'ILC est le plus mature. Élaboré depuis plus de vingt ans, l'ILC dispose d'une preuve de principe technologique avec la source de lumière XFEL, qui exploite un accélérateur linéaire de même type près de Hambourg.

Les usines à Higgs circulaires, conceptuellement similaires au LEP mais au moins dix mille fois plus intenses, nécessitent, en raison des pertes par rayonnement de freinage, un anneau de plus grande circonférence pour atteindre l'énergie nécessaire à la production du boson de Higgs.

Usines à Higgs circulaires et linéaires présentent des performances comparables en ce qui concerne l'étude du boson de Higgs. Les usines circulaires ont un potentiel

\section{Références}

1. European Strategy Group; 2020 Update of the European Strategy for Particle Physics, CERN Council, Genève (2020). https://cds.cern.ch/record/2720129 .

2. Physics Briefing Book: Input for the European Strategy for Particle Physics Update 2020, CERN (2019). https://cds.cern.ch/record/2691414 .

3. European Strategy Group, Deliberation document on the 2020 Update of the European Strategy for Particle Physics, CERN Council, Genève (2020). https://cds.cern.ch/record/2720131.

$4 \cdot \mathrm{L}$. di Ciaccio et G. Hamel de Monchenault, "Découverte du boson de Higgs au LHC ? », Reflets de la physique $\mathbf{3 1}$ (2012) 17-19.

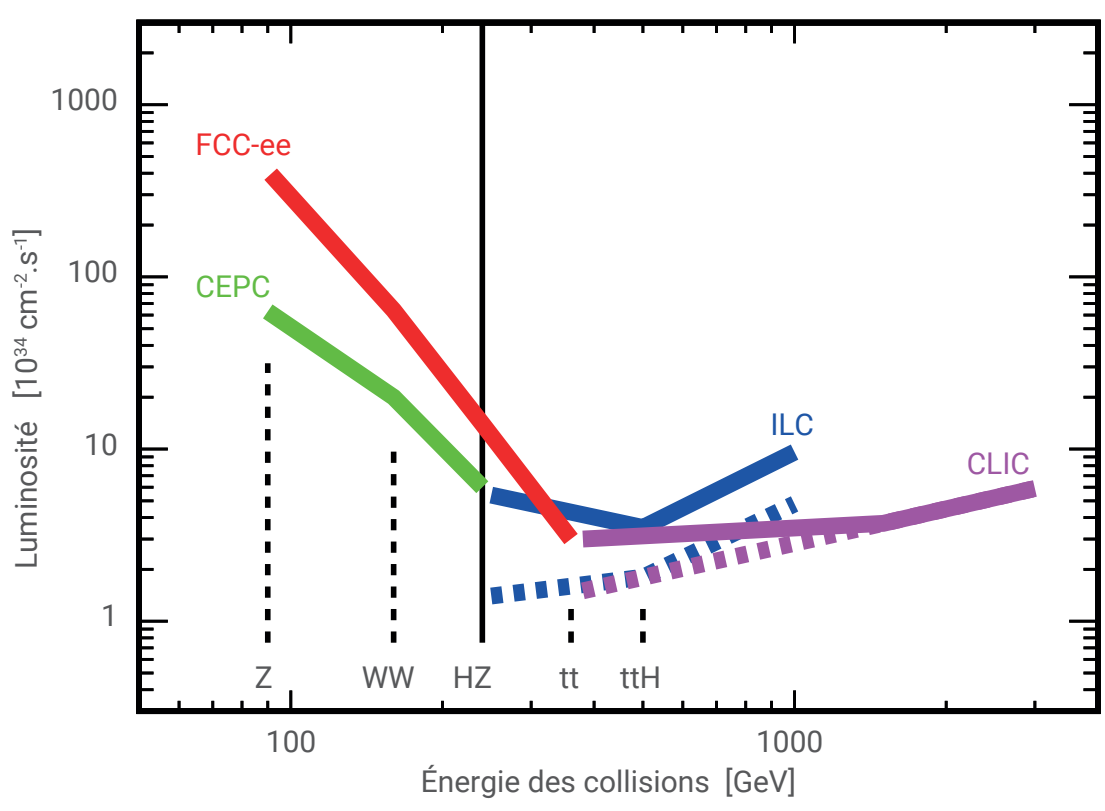

2. La luminosité des quatre projets d'usines à Higgs, exprimée en unités de $10^{34}$ collisions par $\mathrm{cm}^{2}$ et par seconde, est portée en fonction de l'énergie des collisions, exprimée en GeV (d'après [2]). Les échelles sont logarithmiques. Une usine à Higgs est un collisionneur électron-positon capable d'atteindre le seuil de production d'un boson de Higgs accompagné d'un boson Z, indiqué par « $\mathrm{HZ}$ » sur la figure. Les usines circulaires FCC-ee et CEPC fonctionnent à très haute luminosité à l'énergie de la résonance du boson $Z$ (indiquée par « $Z$ ») et au seuil de la production de paires de bosons W (indiquée par "WW ») ; c'est pourquoi on les nomme aussi " usines électrofaibles». La figure suppose deux expériences en deux points d'interaction. Les usines linéaires ILC et CLIC ont le potentiel d'atteindre des énergies élevées, au-delà des seuils de production d'une paire de quarks top ("tt ») et d'un boson de Higgs accompagné d'une paire de quarks top ("ttH »). Pour I'ILC et le CLIC, les courbes en trait pointillé indiquent les performances initiales.

supérieur de mise en défaut des prédictions du MS par l'étude approfondie du secteur électrofaible. Les usines linéaires peuvent être mises à niveau pour explorer de plus hautes énergies, éventuellement pour l'étude de nouvelles particules qui restent à découvrir.

La Stratégie recommande l'intensification de la R\&D sur les technologies d'accélérateurs pour un futur à plus long terme : cela concerne avant tout le développement d'aimants supraconducteurs à haut champ pour collisionneurs de protons, mais aussi des études sur les cavités à haut gradient, l'accélération plasma par champ de sillage, les collisionneurs de muons, les accélérateurs linéaires à récupération d'énergie, etc.

La Stratégie préconise une étude de faisabilité technique et financière pour le collisionneur de protons FCC-hh, qui pourrait voir le jour au CERN dans les dix ans après le terme du HL-LHC. D'une énergie au moins sept fois supérieure à celle du LHC, cette machine nécessite un nouveau tunnel circulaire de grande circonférence (fig. 1) qui pourrait abriter l'usine à Higgs FCC-ee dans un premier temps. Cette étude doit être finalisée avant la prochaine mise à jour de la Stratégie, vers 2026.

La masse non nulle des neutrinos est l'indication la plus convaincante d'une physique au-delà du MS. Les neutrinos jouent un rôle cosmologique majeur dans la formation des structures à grande échelle de l'Univers, et peut-être même dans l'existence de la matière qui nous constitue. C'est pourquoi la Stratégie réitère son soutien aux projets de détecteurs de neutrinos à longue ligne de base aux États-Unis et au Japon, avec une mention pour le projet DUNE aux États-Unis.

La Stratégie insiste sur l'importance de soutenir la théorie, la R\&D sur les détecteurs, les développements logiciels et l'informatique. Elle encourage un programme de diversité scientifique à fort impact, complémentaire de celui des collisionneurs à haute énergie, en soulignant le rôle des 
laboratoires nationaux et en encourageant la participation à des expériences hors d'Europe.

Enfin, la Stratégie appelle de ses vœux des synergies avec les domaines connexes que sont la physique nucléaire et la physique des astroparticules. On y trouve également des recommandations sur la formation de nouvelles générations de jeunes scientifiques, sur le transfert des connaissances et des technologies, sur l'éducation et la communication, ainsi que sur le contrôle de l'impact environnemental de la physique des particules.

\section{Discussion}

L'expertise unique acquise par la construction et l'exploitation du LHC au CERN permet à l'Europe d'occuper la position dominante dans l'exploration de la physique aux plus hautes énergies en collisions de protons. C'est pourquoi il est justifié de placer un collisionneur de protons encore plus puissant que le LHC comme principal objectif à long terme, et de mettre l'accent sur le développement des technologies d'aimants à très haut champ qu'une telle machine nécessite.

Pour l'étape intermédiaire de l'usine à Higgs, l'Europe n'exclut pas de participer à un projet en Asie, si une décision était prise prochainement en ce sens concernant l'ILC ou le CEPC. Le CERN se prépare toutefois à reprendre le flambeau en étudiant la faisabilité de l'usine à Higgs FCC-ee. Reproduisant le schéma fructueux du LHC succédant au LEP dans le même tunnel, le FCC-ee constituerait la première phase du programme FCC se poursuivant sur au moins cinq décennies, avec en point d'orgue le collisionneur de protons aux plus hautes énergies permises par la technologie.

Nul ne peut anticiper avec certitude les prochaines découvertes. L'approche du programme FCC est la plus prometteuse, alliant de façon complémentaire les mesures d'ultra-précision pour tester la théorie du MS en profondeur et l'étude directe de territoires inexplorés à la recherche de nouveaux phénomènes. C'est un projet ambitieux d'ampleur mondiale, avec des retombées technologiques considérables pour la société, dans des domaines comme les aimants supraconducteurs, la cryogénie, le vide, l'électronique et le traitement de quantités gigantesques de données. 